AperTO - Archivio Istituzionale Open Access dell'Università di Torino

Drug delivery nanoparticles in treating chemoresistant tumor cells

This is a pre print version of the following article:

Original Citation:

Availability:

This version is available http://hdl.handle.net/2318/1634214

since 2017-05-16T15:01:02Z

Published version:

DOI:10.2174/0929867323666161205122225

Terms of use:

Open Access

Anyone can freely access the full text of works made available as "Open Access". Works made available under a Creative Commons license can be used according to the terms and conditions of said license. Use of all other works requires consent of the right holder (author or publisher) if not exempted from copyright protection by the applicable law. 
This is the author's final version of the contribution published as:

Barrera, Giuseppina; Daga, Martina; Ferrara, Benedetta; Dianzani, Chiara; Pizzimenti, Stefania; Argenziano, Monica; Cavalli, Roberta; Trotta, Francesco. Drug delivery nanoparticles in treating chemoresistant tumor cells. CURRENT MEDICINAL CHEMISTRY. 23 (999) pp: 1-1.

DOI: $10.2174 / 0929867323666161205122225$

The publisher's version is available at:

http://www.eurekaselect.com/openurl/content.php?genre=article\&doi=10.2174/092986732366616120512222 5

When citing, please refer to the published version.

Link to this full text:

http://hdl.handle.net/2318/1634214 
"Recent Trends in Anticancer Drug Development: Challenges and Opportunities"

\section{Drug delivery nanoparticles in treating chemoresistant tumor cells.}

Giuseppina Barrera ${ }^{1}$, Martina Daga ${ }^{1}$, Benedetta Ferrara ${ }^{2}$, Chiara Dianzani $^{2}$, Stefania Pizzimenti ${ }^{1}$, Monica Argenziano ${ }^{2}$, Roberta Cavalli ${ }^{2}$ and Francesco Trotta ${ }^{3}$.

1. Department of Clinical and Biological Sciences, University of Turin, Corso Raffaello 30, 10125 Turin (Italy).

2. Department of Drug Science and Technology, University of Turin, Via P. Giuria 9, 10125 Turin, Italy,

\section{Department of Chemistry,k University of Turin, Via Pietro Giuria 7, 10125 Turin, Italy}

\section{Abstract}

Intrinsic or acquired chemoresistance represents the main obstacle to the successful treatment of cancer patients. Several mechanisms are involved in multidrug resistance: decreased uptake of hydrophilic drugs, increase of energy dependent efflux, alteration of the redox state, alteration of apoptotic pathways, and modification of the tumor microenvironment. In recent years, several types of nanoparticles have been developed to overcome these obstacles and improve the accumulation and release of drugs at the pathological site. In this review we describe the main mechanisms involved in multidrug resistance and the nanovehicles which have been proposed to target specific aspects of this phenomenon.

\section{Introduction}

Drug resistance, which occurs in nearly all types of cancer, is a major problem in the treatment of cancer patients. Drug resistance can be classified in two ways: the intrinsic resistance, when tumors are resistant prior to treatment, therefore the drugs are not effective even with initial early diagnosis and treatment, and the acquired resistance which occurs after prolonged cycles of chemotherapy, despite an initial positive response [1]. Unfortunately, resistance appears not only to conventional chemotherapy but also to targeted therapies, the so-called ñsmart drugsò, such as kinase inhibitors and tamoxifen that binds to the estrogen receptor [2].

Various mechanisms have been proposed to elucidate pathways and targets of multidrug resistance (MDR) [1]. They can be summarized by three major mechanisms: 1) decreased uptake of hydrophilic drugs, such as folate antagonists, nucleoside analogues and cisplatin, which require transporters to enter cells; 2) various molecular changes in cells, that affect the capacity of cytotoxic drugs to kill cells, including alterations in redox status, increased repair of DNA damage, alteration of apoptotic machinery etc., and 3) increased energy-dependent efflux of hydrophobic drugs, that can easily enter the cells by diffusion through the plasma membrane. This phenomenon occurs 
predominantly via $\mathrm{ABC}$ superfamily transporters and elevated expression levels of these drug efflux pumps [3].

\subsection{ABC Transporter family}

The first mechanism found to explain MDR is the increased efflux of hydrophobic cytotoxic drugs, mediated by members of energy-dependent transporters, the ABC transporter family [4]. The human ATP-binding cassette (ABC) transporters are a large group of membrane protein complexes which consist of 48 members, classified into seven subfamilies from ABC-A through to ABC-G based on their sequence similarities [5]. Among the $48 \mathrm{ABC}$ transporters, the protein complexes located on the plasma membrane significantly affect the intracellular concentration of diverse drugs, drug conjugates and metabolites by export. Several ABC proteins have been characterized to confer resistance to anticancer drugs. Among them, P-glycoprotein (MDR, Pgp or ABCB1), multidrug resistance protein 1 (MRP1 or ABCC1) and ABCG2 (also known as Breast Cancer Resistance Protein: BCRP) are the most frequently associated with MDR [6]. Increased expression of Pgp, as well as of other $\mathrm{ABC}$ proteins, can be induced by exposure of the cells to the drugs, due to genomic mutations or epigenetic modifications of its promoter [7]. The overexpression of these pumps obviously reduce the intracellular concentration of numerous endo- and exo-toxins which are structurally and biochemically distinct, resulting in MDR. To overcome ABC transporter-mediated MDR and sensitize cancer cells to chemotherapeutic agents, some inhibitors of ABC superfamily transporter have been used in association with chemotherapeutic drugs. Although the combined therapies displayed some encouraging clinical results, there is no effective MDR reversing agent approved for an appreciable sensitization of malignant tumors to chemotherapeutic drugs without toxic effects to date. Combined treatment with the first-generation MDR inhibitors such as verapamil and cyclosporine A and anticancer drugs (e.g., mitoxantrone and daunorubicin) led to toxic side effects showing only limited function or no benefits $[8 ; 9]$.

\subsection{Alteration of signaling pathways}

Several signaling pathways have been found to be involved in chemoresistance of cancer cells. A pathway frequently activated, during life of the cancer cells, is the Hedgehog (Hh) pathway [10]. The Hh signaling pathway is one of the important signaling pathways that play key roles in the processes of embryonic development, carcinogenesis, maintenance of cancer stem cells (CSCs), and the acquisition of epithelial-to-mesenchymal transition (EMT) leading to metastasis [10]. The functional transcription activators of the Hh pathway include the GLI proteins. Inhibition of the activity of GLI can interfere with almost all DNA repair types in human cancer and can render tumor cells more vulnerable to lethal DNA damages induced by chemotherapy and radiotherapy [10]. Moreover, the activation of GLI-mediated transcription (through ligand-dependent or ligand- 
independent modes), also induces chemo-resistance also by increasing drug efflux in an ABC transporter-dependent manner [11]. Thus, Hh signaling is an important therapeutic target to overcome MDR and consequently increases the chemotherapeutic response in the treatment of cancer.

Another signaling pathway which has been found to be involved in chemoresistance is the Keap1/Nrf2 pathway [12]. Nrf2 (NF-E2-related factor 2) transcription factor is the master regulator of the antioxidant response of the cells to oxidative stress stimuli through the activation of the synthesis of cytoprotective genes. Under physiological conditions, Nrf2 is present in the cytoplasm where it is bound by Keap1 (Kelch-like ECH-associated protein 1). Keap1 forms a complex with Cul3 and Rbx1, and this E3 ubiquitin ligase complex is able to bind and ubiquitinate Nrf2, resulting in Nrf2 proteasomal degradation [13]. When oxidative stress is present within the cell, the cysteine residues of Keap1 become oxidized, resulting in a conformational change of the Keap1Ï Nrf2 complex which prevents Nrf2 ubiquitination. The stabilized Nrf2 accumulates in nuclei, heterodimerizes with small Maf proteins and activates target genes for cytoprotection through the antioxidant response element (ARE)/electrophile response element (EpRE) [14]. Nrf2 has a dual role in cancer: the canonical protective role in carcinogenesis, and the non-canonical 'dark-side' of Nrf2 in promoting chemoresistance [15]. A Nrf2 role in cisplatin resistance of bladder cancer cells has been indicated by Hayden et al. (2014), which demonstrated that Nrf2 overexpression is associated with clinically relevant cisplatin resistance, that becomes reversible after Nrf2 silencing in experimental models [16]. In ovarian cancer, an aberrant activation of Nrf2 is observed, which confers resistance to cisplatin-induced apoptosis [17].

Increasing evidence has demonstrated the involvement of Yes-associated protein (YAP) with chemoresistance in diverse types of cancers. YAP, a transcriptional co-activator, is a key component of the Hippo tumor-suppressor pathway [18]. Hippo pathway-mediated YAP phosphorylation on Ser127 mainly leads to its cytoplasm sequestration or ubiquitination and degradation [19]. Conversely, unphosphorylated YAP translocates into the nucleus where it binds to the TEAD transcription factor, triggering the expression of several genes involved in organ size control, cell proliferation and survival (i.e. CTGF and survivin) [20]. Indeed, YAP expression inhibition results in reduced cell proliferation and enhanced cell death through modulation of downstream transcriptional targets [21]. Moreover, YAP expression and nuclear localization strongly correlate with poor patient outcome and the progression of several tumors, including bladder and ovarian cancer [22]. In particular, for these two types of tumors, YAP protein has been demonstrated to play a role in cisplatin resistance of cancer cells. Overexpression of Yap2 in immortalized ovarian surface epithelium cells resulted in increased cell proliferation, resistance to 
cisplatin-induced apoptosis, faster cell migration, and anchorage independent growth, while YAP knockdown resulted in increased sensitivity to cisplatin-induced death [23]. Recently it has been demonstrated that constitutive expression and activation of YAP is inversely correlated with ñin vitroò and ñin vivoò cisplatin sensitivity of urothelial cell carcinoma cells [24]. YAP overexpression protects, while YAP knockdown sensitizes cancer cells to chemotherapy and radiation effects via increased accumulation of DNA damage and apoptosis [24].

Through a screening approach, Matz et al (2014) created a library of barcoded pathway-activating mutant complementary DNAs to identify those that enhanced the survival of cancer cells in the presence of 13 clinically relevant, targeted therapies. The Authors found that RAS-MAPK (mitogen-activated protein kinase), Notch1, PI3K (phosphoinositide 3-kinase)-mTOR (mechanistic target of rapamycin), and ER (estrogen receptor) signaling pathways often conferred resistance to specific drugs. In particular, they demonstrated that the activation of the Notch1 pathway promoted acquired resistance to tamoxifen (an ER-targeted therapy) in breast cancer cells and that the inhibition of Notch signaling restored tamoxifen sensitivity. Moreover, Notch1 knockdown fully sensitized drug-resistant melanoma cells to MAPK inhibitors, indicating that, Notch1 signaling may be a therapeutic target in some drug-resistant breast cancers and melanomas [25].

\subsection{Control of redox state.}

The redox status regulation plays an important role in cancer cell survival to the therapy. Many types of cancer cells display a large amount of reactive oxygen species (ROS), due to an aberrant metabolism, mitochondrial dysfunction or activation of oncogenes. This characteristic makes cancer cells more vulnerable to damage by further ROS production induced by exogenous agents [26]. In this context, ROS may exert a cytotoxic effect, leading to the death of malignant cells and thus limiting cancer progression [27]. On the basis of these observations, several ROS-generating agents are currently in clinical trials as single agents or in combination therapy [28]. Alteration of redox status, namely the increase of antioxidant defenses in cancer cells, has been indicated as responsible for radio- and chemoresistance. Indeed, some cancer cells, in particular those in advanced stages of disease, have become highly adapted to intrinsic oxidative stress by upregulating their antioxidant systems [29]. This redox adaptation provides a mechanism of resistance to many anticancer agents, due to increased tolerance of exogenous stress and increased capacity for drug inactivation, mainly linked to the GSH increase [30].

\section{4 miRNA and chemoresistance.}

Several reports have recently highlighted the involvement of endogenous non-coding RNAs, known as microRNAs (miRNAs), in the evolution of drug resistance in cancer cells. MiRNAs are small non-coding RNAs 19 Ï 25 nucleotides in size involved in many biological processes such as 
survival, apoptosis, cell cycle and gene expression regulation [31]. MiRNAs are evolutionarily conserved and work by silencing gene expression. They are involved in many different cancer types and can act as both tumor suppressors and oncogenes [32]. Accumulating evidence is revealing an important role of miRNAs in anticancer drug resistance and their expression profiling can be correlated with the development of resistance [33]. Some miRNAs, such as miRNA 21, have been involved in the resistance toward doxacetal in prostate cancer cells [34] and itôs aberrant expression is critically correlated with the disease stage, drug resistance, and survival of pancreatic cancer patients [35]. The miR-21 is one of the most commonly implicated miRNAs in cancer as its expression is highly up-regulated in a variety of solid tumors, including breast, gastric, colon, lung, pancreatic and ovarian cancers [36]. Several downstream pathways of miR-21 have been identified including phosphatase and tensin homolog (PTEN)/phosphoinositide 3-kinase/protein kinase B (PI3K/Akt), programmed cell death protein 4 (PDCD4, neoplastic transformation inhibitor protein), NF-əB pathways and the HIF-1Ŭpathway, a key downstream target of miR-21 in regulating tumor angiogenesis [37]. Inhibition of miR-21 by curcumin increased pancreatic cancer cell sensitivity to gemcitabine [38]. Roy et al. showed that difluorinated-curcumin decreased miR-21 in 5-FU and oxaliplatin resistant colon cancer cell lines through upregulation of phosphatase and tensin homolog (PTEN) and thus reduced the activity status of the PI3K/Akt pathway [39] which is involved, when activated, in the drug resistance of colon cancer cells [40]. In addition to miRNA 21, other miRNAs, such as, Let 7, miRNA 15, miRNA 16 and miRNA 34, have been found to be involved in chemoresistance [41]. This indicates that miRNA-based therapy may provide a new strategy to overcome drug resistance in future.

\subsection{Tumor microenvironment}

Another aspect involved in chemoresistance is the abnormal tumor microenvironment which induces a collection of cellular stress responses and plays a major role in determining the metabolic status and chemosensitivity in cancer cells [42]. Tumor vasculature is structurally and functionally abnormal, and combined with intrinsically altered tumor cell metabolism, produces heterogeneity in oxygenation, $\mathrm{pH}$, exposure to increased interstitial fluid pressure and the concentrations of glucose and many other metabolites that promote tumor progression and metastatization [43]. For example, in certain microenvironmental contexts, extreme hypoxia causes endoplasmic reticulum stress and activates the unfolded protein response, which provides a further adaptive mechanism that allows tumor cells to survive under adverse metabolic conditions [44]. Moreover, since oxygen is a potent radiosensitizer, hypoxia inhibits effective radiation killing in vitro [45]. and has a direct effect on the effectiveness of drugs, such as mephalan, bleomycin, and etoposide, which require molecular oxygen for maximal efficiency [46]. 
The impairment of the ability of the tumor vasculature to deliver nutrients and remove waste products leads to the increase of environmental acidosis [47], which is involved in chemoresistance too. Indeed, in an acidic extracellular environment, the cellular uptake of some chemotherapeutic drugs, such as doxorubicin, mitoxantrone, or vinblastine, is significantly reduced [48].

In recent years, several types of nanoparticles have been produced in order to overcome the alterations above described which make cancer cells highly resistant to cancer therapy.

\section{Nanoparticles in chemoresistant cells.}

As described above, several mechanisms are involved in induced or intrinsic resistance. Therefore nanomedicine developed drug-loaded nanocarriers able to target specific aspects of chemoresistant cancer cells. In this section we report the more recent issues describing the nanoparticles employed in overcoming the diverse behaviors of chemoresistant tumors.

\subsection{Targeting ABC transporters}

As previously illustrated, the overexpression of $\mathrm{ABCs}$ is a well-known mechanism of MDR in cancer and is associated with therapeutic failure. Since their discovery, ABCs have emerged as attractive therapeutic targets and the search for compounds that inhibit their expression and/or their functional activity has gained growing interest [49]. However, the pharmacological ABC inhibitors present high toxicity and the clinical results have been somewhat disappointing.

Some attempts have been performed to conjugate nontoxic compounds, or lowest doses of toxic compounds, which have been demonstrated an inhibitory activity on some member of ABC transporters, with antineoplatic drugs (Fig.1). In example, Khdair et al. (2009) utilized aerosol OT (AOT)-alginate nanoparticles as a carrier for the simultaneous cellular delivery of methylene blue, an inhibitor of P-gp, and doxorubicin. After photoactivation, nanoparticle-mediated combination therapy resulted in a significant induction of both apoptosis and necrosis in adriamicin-resistant cancer cells compared to single drug treatment [50]. Song et al. (2000) loaded poly(d,1-lactide-coglycolide acid) (PLGA) nanoparticles with vincristine and verapamil hydrochloride (VRP), a calcium channel blocker, able to reverse completely the resistance caused by Pgp in vitro (Huang et al., 1999). Results demonstrated that the co-encapsulation of an anticancer drug and chemosensitizer had high therapeutic effectiveness on MCF-7/ADR breast cancer cells and suggest that this strategy might cause lower normal tissue drug toxicity and fewer drugï drug interactions [51]. Transferrin coated liposomes co-encapsulating verapamil and doxorubicin have been tested by Wu et al (2009), which demonstrated that this association exhibited 5 and 3-fold higher cytotoxicity in doxorubicin-resistant human erythroleukemia K562 cells, compared to non-targeted liposomes and transferrin targeted liposomes with doxorubicin alone, respectively [52]. Other studies have been performed to reduce drug efflux from cancer cells, by utilizing specific platforms for the drug 
delivery. Nanodiamonds (NDs) are promising candidates in this field, demonstrating significant potential as gene/drug delivery platform for cancer therapy. The effectiveness of the ND platform has been demonstrated in daunorubicin delivery in K562 resistant cells [53] and in mitoxantrone delivery in the MDA-MB-231 triple negative breast cancer cell line that was lentivirally transduced for resistance against mitoxantrone [54]. In addition, NDs have been utilized to deliver epirubicin in hepatic cancer stem cells, demonstrating a high effectiveness in overcoming chemoresistance by promoting endocytic uptake and enhancing tumor cell retention [55]. These works demonstrated that ND-drug complexes have favorable drug delivery properties and are capable of improving drug retention and efficacy. Recently, Kovács D. et al. (2015) demonstrated that silver nanoparticles (AgNPs) display an anti-proliferative effect and induce apoptosis mediated cell death both in drug sensitive and in MDR cancer cells and that this action is due to the inhibition of the efflux activity of MDR cancer cells which enhance drug accumulation. Furthermore, AgNPs synergistically potentiate six different antineoplastic agents on drug resistant cells [56].

\subsection{Targeting signal transduction pathways}

Since several signal transduction pathways are activated in cancer cells, some studies have been devoted to investigating whether the delivery of specific inhibitors of a signaling pathway could overcome the MDR. Fan et al. (2010) utilized micellar nanoparticles self-assembled from copolymer folateï chitosan (FAï CS) as carriers to co-deliver doxorubicin and pyrrolidinedithio carbamate (PDTC), an antioxidant and chelator of heavy metals that blocks NF-əB activity by suppressing the release of IəBŬ from NF-əB [57]. These NPs were designed to achieve targeted doxorubicin delivery via endocytosis, with a low $\mathrm{pH}$ responsive endosomal or extracellular drug release, and to overcome resistance via inhibition of NF-əB by PDTC. Results confirmed that the co-delivery of the NF-əB inhibitor PDTC and doxorubicin, effectively overcame drug resistance.

Another approach was the use of metallic nanoparticles to increase the cytotoxic effect of chemotherapeutic drugs. Xiong et al. (2014) have demonstrated that $20 \mathrm{~nm}$ gold nanoparticles (AuNPs) carrying cisplatin prevent cisplatin-induced activation of Akt and NF-kB signaling axis in ovarian cancer cells that are critical for epithelial-mesenchimal transition, stem cell maintenance and drug resistance. In vivo, AuNPs sensitize orthotopically implanted ovarian tumor to a low dose of cisplatin and significantly inhibit tumor growth [58]. Other effects displayed by AuNPs is related to the modification of gene expression as detected in $\mathrm{CaCo} 2$ colon cancer cells by Bajak et al. (2015). The modifications affected some Nrf2 responsive genes (several metallothioneins, HMOX, G6PD, OSGIN1 and GPX2) that were highly up regulated and members of the selenoproteins that were also differentially expressed. These findings indicate that exposure to AuNPs induces 
oxidative stress signaling pathways, and might enhance the anti-cancer properties of chemotherapeutic drugs [59].

The inhibition of the Nrf2 pathway could represent a way to sensitize cancer cells to anticancer drugs by increasing intracellular oxidative stress. Luteolin, as a flavonoid compound, can inhibit Nrf2 and sensitize cancer cells to chemotherapeutic agents. Sabzichi et al. (2014) demonstrated that luteolin loaded in phytosomes, as an advanced nanoparticle carrier, sensitized MDA-MB 231 cells to doxorubicin [60].

\subsection{Targeting redox state}

The extent of antioxidant capacity is actually reported to correlate with the aggressiveness of tumors and it can go beyond the antioxidant capacity of normal cells [61]. In normal cells the glutathione (GSH) concentration is 100 to 1000 times higher than that in the extracellular fluids and circulation and it is further increased in chemoresistant tumor cells. GSH has been recognized as an ideal and ubiquitous internal stimulus for rapid destabilization of nano-carriers inside cells to accomplish efficient intracellular drug release. For this reason GSH-responsive nanoparticles (GSHNPs), which respond to the intracellular concentration of GSH, have been developed [62]. Since in most chemo-resistant and radio-resistant cancer cells the level of intracellular GSH was higher than in the chemo-sensitive or radio-sensitive cancer cells, it has been speculated that GSH-NPs could preferentially drive the drugs in the resistant cancer cells. To pursue this purpose, a new class of $b-$ cyclodextrin GSH-responsive nanosponges (GSH-NSs) that are able to host and to release anticancer drugs in the presence of GSH, at concentrations similar to those found in chemoresistant cancer cells, have been synthesized (Fig.2) [63].

However, even if the antioxidant capacity of chemoresistant cancer cells could constitute a cue for the choice of particles sensitive to the high GSH concentrations, on the other hand, the increase of oxidative stress in this type of cancer cells can contribute to their killing. There is rapidly accumulating evidence sustaining the fact that some types of nanoparticles induce oxidative stress that consequently results in signaling pathway stimulation and apoptotic cell death of cancer cells. Cadmium telluride quantum dots (CdTe-QDs) have recently been shown to effectively induce apoptosis in hepatocellular carcinoma HepG2 cells by activating MAPKs, including JNK, Erk1/2 and p38, as a result of oxidative stress induced in HepG2 cells [64]. Analogously, cadmium sulfide quantum dots (bsCdSQDs) stabilized with a biosurfactant induces ROS-mediated apoptotic cell death in human prostate cancer LNCaP cells [65]. The ROS mediated activation of ERK1/2, JNK and p38 MAPK and apoptosis induction was also observed by treating hepatoma SMMC-7721 cells with cerium oxide nanoparticles. The use of 
ROS scavangers dramatically reduced activated kinases and simultaneously there was a decrease in the apoptotic rate [66]. It has been found that the metal based nanoparticles, such as tungsten carbide-cobalt (WC-Co) nanoparticles, zinc oxide $(\mathrm{ZnO})$ nanoparticles, cobalt nanoparticles, titanium dioxide ( $\mathrm{TiO} 2)$ nanoparticles, nickel oxide nanoparticles, cuprous oxide nanoparticles, silver nanoparticles and zinc nanoparticles can induce oxidative stress and increase the ROS level in a number of different tumor cells [67]. This effect could increase the cytotoxicity of prooxidant cytotoxic drugs.

\subsection{Targeting microenvironmental stressors}

Due to the specific micro environment of the tumor, some of the unique factors such as low $\mathrm{pH}$ and hypoxia can be used as a trigger to overcome MDR. The acidosis in tumor cells, due to the high production of lactate by Warburg effect, and the related acidosis of the environmental tissue due to the impairment of the ability of the tumor vasculature to remove waste products, have been considered for developing of nanoparticles able to release the drug in an acidic environment. Aryal et al. synthetized a Bi(PEG-PLA)-Pt(IV) polymer-cisplatin prodrug conjugate which had a well controlled cisplatin loading yield and showed excellent acid-responsive drug release kinetics, leading to enhanced in vitro cytotoxicity against tumor cells as compared to free cisplatin [68]. Long-circulating and $\mathrm{pH}$-sensitive liposomes containing cisplatin (SpHL-CDDP) have been developed by Leite et al. (2012), which demonstrated that the intravenous administration of SpHLCDDP in solid Ehrlich tumor-bearing mice caused a significant reduction in the tumor volume and a higher tumor growth inhibition ratio with respect to the administration of CDDP alone [69]. Wu et al. (2012) have synthesized mixed micelles of polyethylene glycol based on DSPE-PEG2000, DSPE-PEG3400 and a pH-sensitive polymer PHIS-PEG2000. This mixed micelles showed a pHdependent drug release property with much faster release at around $\mathrm{pH} 5.5$ compared to micelles without PHIS-PEG2000 [70]. Other core-crosslinked pH-sensitive degradable micelles were synthesized based on poly(ethylene glycol)-b-poly(mono-2,4,6-trimethoxy benzylidenepentaerythritol carbonate-co-acryloyl carbonate) (PEG-b-P(TMBPEC-co-AC) copolymer that contains acid-labile acetal and photo-cross-linkable acryloyl groups in the hydrophobic polycarbonate block for intracellular paclitaxel (PTX) release. The in vitro release studies showed that rapid drug release was obtained under mildly acidic conditions, whereas PTX release at $\mathrm{pH} 7.4$ was greatly inhibited [71]. A pH-sensitive mixed copolymer micelles system, composed of hyaluronic acid-g-poly(l-histidine) (HA-PHis) and d-Ǔ-tocopheryl polyethylene glycol 2000 (TPGS2k), an inhibitor of the efflux pumps, was developed to co-deliver doxorubicin and TPGS2k into drug-resistant breast cancer MCF-7 cells. The $\mathrm{pH}$ dependent drug release profile due to the protonation of poly(l-histidine) and the higher cellular uptake conferred to these micelles an 
enhanced MDR reversal effect [72]. Another system to release drug in an acidic environment has been recently proposed by Nogueira et al. (2016), which prepared chitosan-based nanoparticles encapsulating methotrexate modified with the $\mathrm{pH}$-sensitive surfactant 77KS [73]. The presence of $77 \mathrm{KS}$ gives a $\mathrm{pH}$-sensitive behavior to nanoparticles, which allowed accelerated release of methotrexate with decreasing $\mathrm{pH}$ as well as $\mathrm{pH}$-dependent membrane-lytic activity.

As previously described, hypoxia is a characteristic of tumor tissues, which can confer, through hypoxia-inducible factors (HIFs), pro-survival and pro-angiogenetic stimuli. Moreover hypoxia induces macrophage recruitment and transforms them in tumor-associated macrophages (TAMs) which, in turn, promote wound healing, tissue repair and production of anti-inflammatory cytokines like IL-10 [74]. To overcome the hypoxia in the cancer tissue [75] Song et al. (2016) synthesized $\mathrm{MnO}_{2}$ nanoparticles by reducing manganese permanganate $\left(\mathrm{KMnO}_{4}\right)$ to $\mathrm{MnO}_{2}$ with cationic polyelectrolyte poly-(allylamine hydrochloride) (PAH) [76]. The high reactivity of manganese dioxide nanoparticles $\left(\mathrm{MnO}_{2} \mathrm{NPs}\right)$ toward hydrogen peroxide $\left(\mathrm{H}_{2} \mathrm{O}_{2}\right)$, for the simultaneous production of $\mathrm{O}_{2}$ and regulation of $\mathrm{pH}$, alleviates tumor hypoxia. Moreover, the Authors conjugated to $\mathrm{MnO}_{2} \mathrm{NPs}$, hyaluronic acid (HA-MnO2 NPs) which has an immune toxicological effect on macrophages and induces their activation and the production of endogenous ROS, and coated HA$\mathrm{MnO}_{2}$ NPs with mannan that targets the mannose receptor on the surface of TAMs. These modifications further enhanced the ability of $\mathrm{MnO}_{2} \mathrm{NPs}$ to lessen tumor hypoxia and modulate chemoresistance. Indeed, combination treatment of breast tumors with Man-HA-MnO $\mathrm{NPs}_{2}$ and doxorubicin significantly inhibited tumor growth and tumor cell proliferation as compared with chemotherapy alone.

\subsection{Delivery of RNA molecules}

The use of RNA molecules to counteract chemoresistance involves both miRNAs, and small interfering RNA (siRNA). SiRNA are target-specific double-strand RNA molecules synthesized to suppress gene expression through the process of RNA interfering [77]. As previously illustrated, miRNAs are small non-coding RNA molecules (containing about 22 nucleotides) found in plants, animals and some viruses, that functions in RNA silencing and post-transcriptional regulation of gene expression [78]. MiRNAs in cancer can function as oncogenes or tumor suppressors. In cancer, overexpression of some miRNAs, such as miR-21, may promote cancer development by negatively regulating tumor suppressor genes and/or genes that control cell differentiation or apoptosis. On the contrary, underexpression of other miRNAs, such as let-7, function as tumor suppressor genes and may inhibit cancers by regulating oncogenes and/or genes that control cell differentiation or apoptosis [79]. 
The use of miRNA and siRNA in cancer therapy or to counteract chemoresistance is hindered by two main factors: 1) they are unstable in blood since they are substrates of blood nucleases; 2) they have large molecular weights and are hydrophilic, thus they have very poor ability to cross the lipophilic phospholipids bilayers of cell membranes.

To overcome these obstacles, various nanoparticle types have been developed for the delivery miRNA or siRNA into the tumor mass. In the chemoresistance context, siRNA have been utilized to silence genes involved in chemoresistance, whereas the miRNA or anti-miRNAs were utilized to block the action of cellular oncogenes or oncogenic miRNAs, respectively.

\subsubsection{Small interfering RNA delivery}

Although the use of siRNA is an attractive option for post-transcriptional silencing of a target genes, some limitations to clinical application of siRNA drugs in oncology depend on their physicochemical properties, the large molecular weight and polyanionic nature of siRNA which limits its' passive uptake by cells [80]. In addition extracellular barriers exist that prevent an efficient delivery of siRNA and transfection in solid tumors [81]. Moreover, because of plasmatic nucleases, siRNA cannot be directly injected into systemic circulation. Consequently, the encapsulation of siRNA with nanoparticles can shield the siRNA from plasmatic nucleases and immune responses, thus assisting in successful siRNA delivery (Fig. 3).

Lipid-based particles (or liposomes) have been used for decades for the delivery of gene medicines including plasmids, antisense oligonucleotides, and siRNAs. A number of lipid-based particle systems have been developed for delivery of siRNAs or miRNA mimics and used in clinical trials [82]. Wang et al. (2011) demonstrated an increase of siRNA delivery, using lipoplex of siRNA with pegylated cationic liposomes (PCat) [83]. In particular, by targeting survivin, an inducible chemoresistance gene, in combination with paclitaxel treatment, the Authors demonstrated that the silencing of survivin enhanced paclitaxel anticancer activity in a human pancreatic Hs766T xenograft model [84]. However, there are many problems with lipid-based delivery systems in vivo, such as rapid clearance by the liver, lack of target tissue specificity and a low entrapment efficiency [85]. To increase entrapment efficiency, Landen Jr. et al. developed a method of formulating 1,2dioleoyl-sn-glycero-3-phosphatidylcholine-(DOPC-) encapsulated siRNA liposomes [86]. DOPCencapsulated siRNA targeted the oncoprotein EphA2, a tyrosine kinase receptor in the ephrin family, which is highly overexpressed in ovarian cancer and correlates with low response to therapy [87]. This system was highly effective in reducing EphA2 expression 48[t after administration of a single dose in an orthotopic model of ovarian carcinoma [86].

PLGA is a copolymer which is used in a host of Food and Drug Administration (FDA) approved therapeutic devices, owing to its biodegradability and biocompatibility. Recently PLGA is being 
used as a nanocarrier for plasmid DNA and siRNA delivery. The advantages conferred by PLGAbased siRNA delivery include high plasma stability and endocytic uptake [88]. Modification of PLGA leading to the targeting of specific aspects of resistant tumors can increase the accumulation of these particles in tumor sites. For example pH-labile linkage-bridged block copolymer of poly(ethylene glycol) with poly(lacide-co-glycolide) (PEG-Dlinkm-PLGA) was used for siRNA delivery. The obtained siRNA-encapsulating PEG-Dlinkm-PLGA nanoparticle gained efficiently prolonged circulation in the blood and preferential accumulation in tumor sites via the PEGylation [89]. Recent studies have shown that the suppression of gene products involved in the DNA repair pathway, such as REV1/REV31 can sensitize intrinsically resistant tumors to chemotherapy and reduce the frequency of acquired drug resistance of relapsed tumors. Thus, a combination of conventional DNA-damaging chemotherapy with siRNA-based therapeutics has been proposed by $\mathrm{Xu}$ et al., (2013). The Authors found that nanoparticles (NPs) self-assembled from biodegradable PLGA-PEG block copolymers delivering a cisplatin prodrug in combination with REVI/REV $3 L$ specific siRNAs revealed a synergistic effect on tumor inhibition in a human lymph node carcinoma of the xenograft mouse model and they were strikingly more effective than platinum monotherapy [90].

Chitosan-encapsulated TWIST-siRNA nanoparticles were constructed and used to silence the TWIST gene [91], which has been involved in chemoresistance and poor prognosis of nasopharyngeal carcinoma [92]. It has been shown that nanoparticles successfully knock-down TWIST expression in a human nasopharyngeal cell line (CNE2), and significantly sensitized CNE2 cells to irradiation.

\subsection{2 miRNA delivery}

MiRNAs play critical roles in modulating the oncogenic driver pathways involved in the acquisition of resistance to cancer treatments. Despite promising results in the development of miRNA therapeutics and successes on in vitro studies, limited progress has been made with in vivo studies or clinical trials. To increase the resistance to serum nuclease, avoid the activation of the innate immune system, and reduce off-target effects, chemical modifications of miRNA molecules [93] and different types of nano-vehicles have been proposed to treat different cancer types [94].

Triple negative breast cancers (TNBCs) are a specific subtype of epithelial breast tumours that are immunohistochemically negative for the protein expression of the estrogen receptor (ER), the progesterone receptor (PR) and lack overexpression/gene amplification of HER2 [95]. Treatment of TNBC with chemotherapeutics such as taxanes is initially very effective in most patients. However, the majority of these tumors develop resistance [96]. To treat and overcome resistance of TNBC several nano-vehicles have been proposed. Wang et al. (2015) have loaded hyaluronic acid (HA)- 
decorated polyethylenimine-poly(d,1-lactide-co-glycolide) (PEI-PLGA) nanoparticles with doxorubicin and miR-542-3p, a potent tumor suppressor molecule, which targets tumor suppressor p53 and apoptosis inhibitor survival [97]. The co-delivery of doxorubicin and miR-542-3p increased both drug uptake and cytotoxicity in triple negative breast cancer cells.

Recently an hydrophilic poly(ethylene glycol)-conjugated poly(lactic-co-glycolic acid) nanoparticle (PLGA-PEG-NP) delivery system has been demonstrated the ability to successfully deliver antisense-miR-21. miR-21 is an oncogenic miRNA involved in tumor initiation, progression, invasion and metastasis in several cancers, including triple negative breast cancer (TNBC). Antisense-miR-21-loaded nanoparticles (NPs) were able to increase the apoptoic effect of orlistat-loaded NPs in triple negative breast cancer cells [98].

An important finding in systemic delivery of anti-miRNA has been reported by Shu D. et al. (2015) which proposed an application of RNA nanotechnology for specific and efficient delivery of anti-miR-21 to block the growth of triple negative breast cancer in orthotopic mouse models [99]. These therapeutic RNA nanoparticles contain an 8-nt sequence complementary to the seed region of miR-21, and a 39-nt sequence complementary to epidermal growth factor receptor (EGFR). They simultaneously target EGFR for internalizing RNA nanoparticles into cancer cells via receptor mediated endocytosis and are able to inhibit miR- 21 activity. In addition, these particles demonstrate a resistance toward RNase and are thermodynamically stable, thus remaining intact after systemic injection into mice.

Another tumor which displays a great resistance to chemotherapic treatment is glioblastoma. Despite important advances in cancer treatment, which resulted in significant improvement of clinical outcomes, glioblastoma relapse is very frequent and patient survival is 12 to 15 months after diagnosis [100]. Successful in vivo delivery of anti-miRNA oligonucleotides to brain tumors requires the carriers not only possessing bioavailability but also overcoming the bloodï brain barrier and enhance target cell uptake, while sparing the normal tissues. In this regard, Costa et al. (2015) proposed stable nucleic acid lipid particles (SNALPs) coupled with chlorotoxin (CTX), a scorpion-derived peptide that was reported as a reliable and specific marker for gliomas [101] to the surface of stabilized liposomes, for delivery of anti-miR-21 oligonucleotides to glioblastoma cells [102]. This delivery system enhanced uptake in brain tumors and increased miR-21 silencing, while showing no signs of systemic immunogenicity. Moreover, the systemic treatment with targeted nanoparticle-formulated anti-miR-21 oligonucleotides and sunitinib (a tyrosine kinase inhibitor) decreased tumor cell proliferation and tumor size and enhanced apoptosis in glioblastomabearing mice.

\subsection{Co-delivery of chemotherapeutic drugs}

Multi-targeted strategies are necessary to overcome multidrug resistance mechanisms and several studies have investigated effective combinatorial approaches for cancer treatments. The use of gemcitabine (2NGAlifluorodeoxycytidine; $\mathrm{dFdC}$ ) in combination with carboplatin to treat patients with advanced, refractory, or recurrent ovarian cancer as well as patients who showed initial 
resistance to platin-based treatments has been recently approved by the U.S. Food and Drug Administration (FDA) [103]. However, cancer cells often show a defective transport of gemcitabine. To overcome the transporter defects in ovarian cancer cells, Hung et al. (2015) constructed NPs from poly(d,l-lactic-co-glycolic acid)-block (PLGA-b)-poly(ethylene glycol) (PEG) polymer (PLGA- $b$-PEG-OH) containing gemcitabine, cisplatin or both compounds [104]. and demonstrated that this construct highly increased the chemotherapeutic efficacy of gemcitabine. Moreover, the delivery of a gemcitabine-cisplatin combination in such nanoparticle formulation increased their synergistic interactions.

Magnetite doped mesoporous silica nanoparticles (MSNs), in which both internal porous and external surface of MSN were respectively exploited to load two different kinds of cytotoxic agents, camptothecin and arsenic trioxide, have been prepared by Muhammad Fet al. (2014) [105]. MSNs were used to inhibit proliferation of BxPC-3 pancreatic cancer cells, which are associated with a low responsiveness to conventional chemotherapies. Results obtained (da molti autori, ci sono moltissimi lavori in letteratura) demonstrated that the cell inhibition performance of dual drug nanoformulation was significantly higher than single drug formulation, possibly due to additional or synergistic effects.

\section{Conclusion and future prospective}

Studies on the mechanisms involved in chemoresistance are progressing rapidly and new targets for the nanomedicine application are continuously being found. In addition, advancement in the field of nanomedicine has led to the development of several types of nanoparticles able to overcome multidrug resistance mechanisms and re-sensitize cancer cells to the anticancer drug. Multivalent constructs may include both drugs acting on cancer cells in combination with inhibitors of drug efflux, or siRNA against genes involved in specific pathways, in combination with anticancer drugs. Results obtained have demonstrated that nano-drug delivery systems are a versatile platform for delivery of anticancer drugs and for overcoming cancer drug resistance mechanisms, maximizing chemotherapeutic efficacy. However, many multifunctional nano-platforms are still in the initial stage of development and a number of safety issues and therapeutic efficacy issues of the nanomaterials should also be addressed before they enter into clinical trials. During the transition ñbenchtop-to-clinicò is also necessary to increase manufacturing reproducibility and overcome the lack of collaboration in innovative research between academia and the pharmaceutical industry.

\section{References}


[1] Rebucci, M.; Michiels, C. Molecular aspects of cancer cell resistance to chemotherapy. Biochem. Pharmacol., 2013, 85, 1219-26.

[2] Raguz, S.; Yague, E. Resistance to chemotherapy: new treatments novel insights into an old problem. Br. J. Cancer, 2008, 99, $387 і ̈ 391$.

[3] Szakács, G.; Paterson, J.K.; Ludwig, J.A.; Booth-Genthe, C.; Gottesman, M.M. Targeting multidrug resistance in cancer. Nat. Rev. Drug. Discov., 2006, 5(3), 219-34.

[4] Gottesman, M.M.; Fojo, T; Bates, S.E. Multidrug resistance in cancer: role of ATP-dependent transporters. Nat. Rev. Cancer, 2002, 2, $48 і ̈ 58$.

[5] Ween, M.P; Armstrong, M.A.; Oehler, M.K.; Ricciardelli, C. The role of ABC transporters in ovarian cancer progression and chemoresistance. Crit. Rev. Oncol. Hematol, 2015, 96, 220-256.

[6] Wu, C.P.; Hsieh, C.H.; Wu, Y.S. The emergence of drug transporter-mediated multidrug resistance to cancer chemotherapy. Mol. Pharm., 2011, 8, 1996ï 2011.

[7] Chen, K.G.; Sikic, B.I. Molecular pathways: regulation and therapeutic implications of multidrug resistance. Clin. Cancer Res., 2012, 18, $1863 і ̈ 1869$.

[8] Daenen, S.; van der Holt, B.; Verhoef, G.E.; Lowenberg, B.; Wijermans, P.W.; Huijgens, P.C. et al. Addition of cyclosporin A to the combination of mitoxantrone and etoposide to overcome resistance to chemotherapy in refractory or relapsing acute myeloid leukaemia: a randomised phase II trial from HOVON, the Dutch-Belgian Haemato-Oncology Working Group for adults. Leuk. Res., 2004, 28, $1057 і ̈ 1067$.

[9] List, A.F.; Kopecky, K.J.; Willman, C.L.; Head, D.R.; Persons, D.L.; Slovak, M.L. et al. Benefit of cyclosporine modulation of drug resistance in patients with poor-risk acute myeloid leukemia: a Southwest Oncology Group study. Blood, 2001, 98, 3212 ï 3220. 
[10] Meng, E.; Hanna, A.; Samant, R.S.; Shevde, L.A. The Impact of Hedgehog Signaling Pathway on DNA Repair Mechanisms in Human Cancer. Cancers, 2015, 7(3), 1333-48.

[11] Shevde, L.A.; Samant, R.S. Non-classical hedgehog-GLI signaling and its clinical implications. Int. J. Cancer, 2014, 135, 1 ï 6.

[12] Furfaro, A.L.; Traverso, N.; Domenicotti, C.; Piras, S.; Moretta, L.; Marinari, U.M.; Pronzato, M.A.; Nitti, M. The Nrf2/HO-1 Axis in Cancer Cell Growth and Chemoresistance. Oxid. Med. Cell. Longev., 2016, 1958174.

[13] Taguchi, K.; Motohashi, H.; Yamamoto, M. Molecular mechanisms of the Keap1Ï Nrf2 pathway in stress response and cancer evolution. Genes Cells, 2011, 16, 123-40.

[14] Itoh, K.; Tong, K.I.; Yamamoto, M. Molecular mechanism activating Nrf2-Keap1 pathway in regulation of adaptive response to electrophiles. Free Radic. Biol. Med., 2004, 36, $1208 і ̈ 1213$.

[15] No, J.H.; Kim, Y.B.; Song, Y.S. Targeting nrf2 signaling to combat chemoresistance. J. Cancer Prev., 2014, 19, 111-7.

[16] Hayden, A.; Douglas, J.; Sommerlad, M.; Andrews, L.; Gould, K.; Hussain, S.; Thomas, G.J.; Packham, G.; Crabb, S.J. The Nrf2 transcription factor contributes to resistance to cisplatin in bladder cancer. Urol. Oncol., 2014, 32, 806-14.

[17] Van der Wijst, M.G.; Brown, R.; Rots, M.G. Nrf2, the master redox switch: the Achilles' heel of ovarian cancer? Biochim. Biophys. Acta., 2014, 1846, 494-509.

[18] Harvey, K.F.; Zhang, X.; Thomas, D.M. The Hippo pathway and human cancer. Nat. Rev. Cancer, 2013, 13, $246 і ̈ 257$.

[19] Zhao, B.; Li, L.; Tumaneng, K.; Wang, C.Y.; Guan, K.L. A coordinated phosphorylation by Lats and CK1 regulates YAP stability through SCF (beta-TRCP). Genes Dev., 2010, 24, 721 ï 85. 
[20] Zhao, B.; Ye, X.; Yu, J.; Li, L.; Li, W.; Li, S.; Yu, J.; Lin, J.D.; Wang, C.Y.; Chinnaiyan, A.M.; Lai, Z.C.; Guan, K.L. TEAD mediates YAP-dependent gene induction and growth control. Genes Dev., 2008, 22, $1962 і ̈ 1971$.

[21] Steinhardt, A.A.; Gayyed, M.F.; Klein, A.P.; Dong, J.; Maitra, A.; Pan, D.; Montgomery, E.A.; Anders, R.A. Expression of Yes-associated protein in common solid tumors. Hum. Pathol., 2008, $39,1582-9$.

[22] Fernandez-L, A.; Kenney, A.M. The Hippo in the room: a new look at a key pathway in cell growth and transformation. Cell Cycle, 2010, 9, 2292-9.

[23] Hall, C.A.; Wang, R.; Miao, J.; Oliva, E.; Shen, X.; Wheeler, T.; Hilsenbeck, S.G.; Orsulic, S.; Goode, S. Hippo pathway effector YAP is an ovarian cancer oncogene. Cancer Res., 2010, 70, 8517-25.

[24] Ciamporcero, E.; Shen, H.; Ramakrishnan, S.; Yu Ku, S.; Chintala, S.; Shen, L.; Adelaiye, R.; Miles, K.M.; Ullio, C.; Pizzimenti, S.; Daga, M.; Azabdaftari, G.; Attwood, K.; Johnson, C.; Zhang, J.; Barrera, G.; Pili, R. YAP activation protects urothelial cell carcinoma from treatment-induced DNA damage. Oncogene, 2015, 35, 1541-1553.

[25] Martz, C.A.; Ottina, K.A.; Singleton, K.R. et al. Systematic identification of signaling pathways with potential to confer anticancer drug resistance. Sci. Signal., 2014, 7, ra121.

[26] Pelicano, H.; Carney, D.; Huang, P. ROS stress in cancer cells and therapeutic implications. Drug Resist., 2004, 7, 97-110.

[27] Fruehauf, J. P.; Meyskens, F. L., Jr. Reactive oxygen species: a breath of life or death? Clin. Cancer Res., 2007, 13, 789-794.

[28] Cabello, C. M.; Bair, W. B., 3rd; Wondrak, G. T. Experimental therapeutics: targeting the redox Achilles heel of cancer. Curr. Opin. Investig. Drugs, 2007, 8, 1022-1037.

[29] Barrera, G. Oxidative stress and lipid peroxidation products in cancer progression and therapy. ISRN Oncol., 2012, 201, 137289. 
[30] Trachootham, D.; Alexandre, J.; Huang, P. Targeting cancer cells by ROS-mediated mechanisms: a radical therapeutic approach? Nat. Rev. Drug Discov,. 2009, 8, 579-591.

Bartel, D.P. MicroRNAs: genomics, biogenesis, mechanism, and function. Cell, 2004, 116, 281-97.

[31] Calin, G.A.; Croce, C.M. MicroRNA signatures in human cancers. Nat. Rev. Cancer, 2006, 6, 857-66.

[32] Ventura, A.; Jacks, T. MicroRNAs and cancer: short RNAs go a long way. Cell, 2009, 136, 586-91.

[33] Kutanzi, K.R. et al. MicroRNA-mediated drug resistance in breast cancer Clin. Epigenetics, 2011, 2, $171 \ddot{1} 185$.

[34] Kopczyథkka, E. Role of microRNAs in the resistance of prostate cancer to docetaxel and paclitaxel. Contemp. Oncol., 2016, 19(6), 423-7.

[35] Li, Y.; Sarkar, F.H. MicroRNA Targeted Therapeutic Approach for Pancreatic Cancer. Int. J. Biol. Sci., 2016, 12(3), 326-37.

[36] Si, M.L.; Zhu, S.; Wu, H. et al. miR-21-mediated tumor growth. Oncogene, 2007, 26, 2799803.

[37] Liu, L.Z.; Li, C.; Chen, Q. MiR-21 induced angiogenesis through AKT and ERK activation and HIF-1a expression. PLoS One, 2011, 6, 1 Ï 9.

[38] Ali, S.; Ahmad, A.; Banerjee, S. et al. Gemcitabine sensitivity can be induced in pancreatic cancer cells through modulation of miR-200 and miR-21 expression by curcumin or its analogue CDF. Cancer Res., 2010, 70, 3606-17.

[39] Roy, S.; Yu, Y.; Padhye, S.B. et al. Difluorinated-curcumin (CDF) restores PTEN expression in colon cancer cells by down-regulating miR-21. PLoS One, 2013, 8, e68543h. 
[40] Chen, J.; Huang, X.F.; Qiao, L. et al. Insulin caused drug resistance to oxaliplatin in colon cancer cell line HT29. J. Gastrointest. Oncol., 2011, 2, 27-33.

[41] Dehghanzadeh, R.; Jadidi-Niaragh, F.; Gharibi, T.; Yousefi, M. MicroRNA-induced drug resistance in gastric cancer. Biomed. Pharmacother., 2015, 74, 191-9.

[42] Cairns, R.A.; Harris, I.S.; Mak, T.W. Regulation of cancer cell metabolism. Nature Reviews Cancer, 2011, 11, 85ï 95.

[43] Lunt, S. J..; Chaudary, N. \& Hill, R. P. The tumor microenvironment and metastatic disease. Clin. Exp. Metastasis, 2009, 26, 19 ï 34.

[44] $\mathrm{Bi}, \mathrm{M}$. et al. ER stress-regulated translation increases tolerance to extreme hypoxia and promotes tumor growth. EMBO J., 2015, 24, 3470ї 3481.

[45] Mohindra, J.K.; Rauth, A.M. Increased cell killing by metronidazole and nitrofurazone of hypoxic compared to aerobic mammalian cells. Cancer Res., 1976, 36, 930 ï 6

[46] Koch, S.; Mayer, F.; Honecker, F.; Schittenhelm, M.; Bokemeyer, C. Efficacy of cytotoxic agents used in the treatment of testicular germ cell tumours under normoxic and hypoxic conditions in vitro. Br. J. Cancer, 2003, 89, 2133 Ï 9.

[47] Griffiths, J.R.; McIntyre, D.J.; Howe, F.A.; Stubbs, M. Why are cancers acidic? A carriermediated diffusion model for $\mathrm{H}+$ transport in the interstitial fluid. Novartis Found Symp, 2001; 240, 46ї 62; discussion 62ï 47, $152 і ̈ 3$.

[48] Mahoney, B.P.; Raghunand, N.; Baggett, B.; Gillies, R.J. Tumor acidity, ion trapping and chemotherapeutics. I. Acid $\mathrm{pH}$ affects the distribution of chemotherapeutic agents in vitro. Biochem. Pharmacol., 2003, 66, $1207 i ̈ 18$.

[49] Mimeault, M.; Batra, S.K. Potential molecular therapeutic targets in cancer stem/progenitor cells: are ATP-binding cassette membrane transporters appropriate targets to eliminate cancerinitiating cells? In: Stem Cell Biology in Health and Disease; T. Dittmar, K.S. Zanker, Eds.; Springer Science + Business Media B.V: Dordrecht, 2010; Vol. 17, pp. $3851 ̈ 421$. 
[50] Khdair, A.; Handa, H.; Mao, G.; Panyam, J. Nanoparticle-mediated combination chemotherapy and photodynamic therapy overcomes tumor drug resistance in vitro. Eur. J. Pharm. Biopharm., 2009, 71, 214ї 222.

[51] Song, X.; Cai, Z.; Zheng, Y.; He, G.; Cui, F.; Gong, D. et al. Reversion of multidrug resistance by co-encapsulation of vincristine and verapamil in PLGA nanoparticles. Eur. J. Pharm. Sci., 2009, 37, $300 і ̈ 305$.

[52] Wu, J.; Lu, Y.; Lee, A.; Pan, X.; Yang, X.; Zhao, X. et al. Reversal of multidrug resistance by transferrin-conjugated liposomes co-encapsulating doxorubicin and verapamil. J. Pharmacol. Pharm. Sci., 2007, 10, 350 ï 357.

[53] Man, H.B.; Kim, H.; Kim, H.J.; Robinson, E.; Liu, W.K.; Chow, E.K.; Ho, D. Synthesis of nanodiamond-daunorubicin conjugates to overcome multidrug chemoresistance in leukemia. Nanomedicine, 2014, 10(2), 359-69.

[54] Toh, T.B.; Lee, D.K.; Hou, W.; Abdullah, L.N.; Nguyen, J.; Ho, D.; Chow, E.K. Nanodiamond-mitoxantrone complexes enhance drug retention in chemoresistant breast cancer cells. Mol. Pharm., 2014, 11(8), 2683-91.

[55] Wang, X.; Low, X.C.; Hou, W.; Abdullah, L.N.; Toh, T.B.; Mohd Abdul Rashid, M.; Ho, D.; Chow, E.K. Epirubicin-adsorbed nanodiamonds kill chemoresistant hepatic cancer stem cells. ACS Nano., 2014, 8(12), 12151-66.

[56] Kovács, D.; SzṚke, K.; Igaz, N.; Spengler, G.; Molnár, J.; Tóth, T.; Madarász, D.; Rázga, Z.; Kónya, Z.; Boros, I.M.; Kiricsi, M. Silver nanoparticles modulate ABC transporter activity and enhance chemotherapy in multidrug resistant cancer. Nanomedicine, 2016, 12(3), 601 ï 610.

[57] Fan, L.; Li, F.; Zhang, H.T.; Wang, Y.K.; Cheng, C.; Li, X.Y. et al. Co-delivery of PDTC and doxorubicin by multifunctional micellar nanoparticles to achieve active targeted drug delivery and overcome multidrug resistance. Biomaterials, 2010, 31, 5634ї 5642. 
[58] Xiong, X.; Arvizo, R.R.; Saha, S.; Robertson, D.J.; McMeekin, S.; Bhattacharya, R.; Mukherjee, P. Sensitization of ovarian cancer cells to cisplatin by gold nanoparticles. Oncotarget, 2014, 5(15), 6453-65.

[59] Bajak, E.; Fabbri, M.; Ponti, J.; Gioria, S.; Ojea-Jiménez, I.; Collotta, A.; Mariani, V.; Gilliland, D.; Rossi, F.; Gribaldo, L. Changes in Caco-2 cells transcriptome profiles upon exposure to gold nanoparticles. Toxicol. Lett., 2015, 233(2), 187-99.

[60] Sabzichi, M.; Hamishehkar, H.; Ramezani, F.; Sharifi, S.; Tabasinezhad, M.; Pirouzpanah, M.; Ghanbari, P.; Samadi, N. Luteolin-loaded phytosomes sensitize human breast carcinoma MDA-MB 231 cells to doxorubicin by suppressing Nrf2 mediated signalling. Asian. Pac. J. Cancer Prev., 2014, 15(13), 5311-6.

[61] Kobayashi, C.I.; Suda, T. Regulation of reactive oxygen species in stem cells and cancer stem cells. J. Cell. Physiol., 2012, 227(2), 421-30.

[62] Cheng, R.; Feng, F.; Meng, F.; Deng, C.; Feijen, J.; Zhong, Z. Glutathione-responsive nanovehicles as a promising platform for targeted intracellular drug and gene delivery. J. Control Release, 2011, 152, 2-12.

[63] Trotta, F.; Caldera, F.; Dianzani, C.; Argenziano, M.; Barrera, G.; Cavalli, R.

Glutathione Bioresponsive Cyclodextrin Nanosponges. Chempluschem. DOI: 10.1002/cplu.201500531.

[64] Nguyen, K.C.; Willmore, W.G.; Tayabali, A.F. Cadmium telluride quantum dots cause oxidative stress leading to extrinsic and intrinsic apoptosis in hepatocellular carcinoma HepG2 cells. Toxicology, 2013, 306, 114-23.

[65] Singh, B.R.; Singh, B.N.; Khan, W.; Singh, H.B.; Naqvi, A.H. ROS-mediated apoptotic cell death in prostate cancer LNCaP cells induced by biosurfactant stabilized $\mathrm{CdS}$ quantum dots. Biomaterials, 2012, 33, 5753-67. 
[66] Cheng, G.; Guo, W.; Han, L. et al. Cerium oxide nanoparticles induce cytotoxicity in human hepatoma SMMC-7721 cells via oxidative stress and the activation of MAPK signaling pathways. Toxicol. In Vitro, 2013, 27, 1082-8.

[67] Nogueira, D.R.; Rolim, C.M.; Farooqi, A.A. Nanoparticle induced oxidative stress in cancer cells: adding new pieces to an incomplete jigsaw puzzle. Asian. Pac. J. Cancer Prev., 2014, 15(12), 4739-43.

[68] Aryal, S.; Hu, C.M.; Zhang, L. Polymer--cisplatin conjugate nanoparticles for acid-responsive drug delivery. ACS Nano., 2010, 4(1), 251-8.

[69] Leite, E.A.; Souza, C.M.; Carvalho-Júnior, A.D.; Coelho, L.G.; Lana, A.M.; Cassali, G.D.; Oliveira, M.C. Encapsulation of cisplatin in long-circulating and $\mathrm{pH}$-sensitive liposomes improves its antitumor effect and reduces acute toxicity. Int. J. Nanomedicine, 2012, 7, 5259-69.

[70] Wu, H.; Zhu, L.; Torchilin, V.P. pH-sensitive poly (histidine)-PEG/DSPE-PEG co-polymer micelles for cytosolic drug delivery. Biomaterials, 2013, 34, 1213ï 1222.

[71] Wu, Y.; Chen, W.; Meng, F.; Wang, Z.; Cheng, R.; Deng, C.; Liu, H.; Zhong, Z. Corecrosslinked $\mathrm{pH}$-sensitive degradable micelles: A promising approach to resolve the extracellular stability versus intracellular drug release dilemma. J. Control Release, 2012, 164(3), 338-45.

[72] Qiu, L.; Qiao, M.; Chen, Q.; Tian, C.; Long, M.; Wang, M. et al. Enhanced effect of pHsensitive mixed copolymer micelles for overcoming multidrug resistance of doxorubicin. Biomaterials, 2014, 35, 9877 ï 9887.

[73] Nogueira, D.R.; Scheeren, L.E.; Macedo, L.B.; Marcolino, A.I.; Pilar Vinardell, M.; Mitjans, M.; Rosa Infante, M.; Farooqi, A.A.; Rolim, C.M. Inclusion of a pH-responsive amino acid-based amphiphile in methotrexate-loaded chitosan nanoparticles as a delivery strategy in cancer therapy. Amino Acids, 2016, 48(1), 157-68.

[74] Hao, N. B.; Lu, M. H.; Fan, Y. H.; Cao, Y. L.; Zhang, Z. R.; Yang, S. M. Macrophages in Tumor Microenvironments and the Progression of Tumors. Clin. Dev. Immunol., 2012, 948098. 
[75] Martinez, F. O.; Sica, A.; Mantovani, A.; Locati, M. Macrophage Activation and Polarization. Front. Biosci., 2008, 13, 453ī 461.

[76] Song, M.; Liu, T.; Shi, C.; Zhang, X.; Chen, X. Bioconjugated Manganese Dioxide Nanoparticles Enhance Chemotherapy Response by Priming Tumor-Associated Macrophages toward M1-like Phenotype and Attenuating Tumor Hypoxia. ACS Nano., 2016, 10(1), 633-47.

[77] Elbashir, S.M. et al. Duplexes of 21-nucleotide RNAs mediate RNA interference in cultured mammalian cells. Nature, 2001, 411, $494 і ̈ 498$.

[78] Ambros, V. The functions of animal microRNAs. Nature, 2004, 431(7006), 350 ï 5.

[79] Zhang, B.; Pan, X.; Cobb, G.P.; Anderson, T.A. microRNAs as oncogenes and tumor suppressors. Dev. Biol., 2007, 302(1), 1-12.

[80] Young, S.W.; Stenzel, M.; Jia-Lin, Y. Nanoparticle-siRNA: A potential cancer therapy? Crit. Rev. Oncol. Hematol., 2016, 98, 159-69.

[81] Zhang, Y.; Satterlee, A.; Huang, L. In vivo gene delivery by nonviral vectors: overcoming hurdles? Mol. Ther., 2012, 20(7), $1298 i ̄ 304$.

[82] Shields, N.; Dodd, K. J.; Abblitt, C. Do children with Down Syndrome perform sufficient physical activity to maintain good health? A pilot study. Adapt. Phys. Act. Q., 2009, 26(4), 307ī 20

[83] Wong, H.L.; Shen, Z.; Lu, Z.; Wientjes, M.G.; Au, J.L. Paclitaxel tumor-priming enhances siRNA delivery and transfection in 3-dimensional tumor cultures. Mol. Pharm., 2011, 8, 833ï 840.

[84] Wang, J.; Lu, Z.; Wang, J.; Cui, M.; Yeung, B.Z.; Cole, D.J.; Wientjes, M.G.; Au, J.L. Paclitaxel tumor priming promotes delivery and transfection of intravenous lipid-siRNA in pancreatic tumors. J. Control Release, 2015, 216, 103-10.

[85] Wu, S.Y.; McMillan, N.A.J. Lipidic systems for in vivo siRNA delivery. AAPS Journal, 2009, 11(4), 639ї 652. 
[86] Landen, C.N.Jr., Chavez-Reyes, A.; Bucana, C. et al. Therapeutic EphA2 gene targeting in vivo using neutral liposomal small interfering RNA delivery. Cancer Research, 2005, 65(15), $6910 і ̈ 6918$.

[87] Thaker, P.H.; Deavers, M.; Celestino, J. et al. EphA2 expression is associated with aggressive features in ovarian carcinoma. Clin. Cancer Res., 2004, 10, $5145 \mathrm{Ï} 50$.

[88] Lee, J.M.; Yoon, T.J.; Cho, Y.S. Recent developments in nanoparticle-based siRNA delivery for cancer therapy. BioMed. Res. Int., 2013, p. 10.

[89] Xu, C.F.; Zhang, H.B.; Sun, C.Y.; Liu, Y.; Shen, S.; Yang, X.Z.; Zhu, Y.H.; Wang, J. Tumor acidity-sensitive linkage-bridged block copolymer for therapeutic siRNA delivery. Biomaterials, 2016, $88,48-59$.

[90] Xu, X.; Xie, K.; Zhang, X.Q.; Pridgen, E.M.; Park, G.Y.; Cui, D.S.; Shi, J.; Wu, J.; Kantoff, P.W.; Lippard, S.J.; Langer, R.; Walker, G.C.; Farokhzad, O.C. Enhancing tumor cell response to chemotherapy through nanoparticle-mediated codelivery of siRNA and cisplatin prodrug. Proc. Natl. Acad. Sci. USA, 2013, 110(46), 18638-43.

[91] Zhuo, X.; Chang, A.; Huang, C.; Yang, L.; Zhao, H.; Wu, Y.; Zhou, Q. Nanoparticle-mediated down-regulation of TWIST increases radiosensitivity of nasopharyngeal carcinoma cells via ERK pathway. Am. J. Cancer Res., 2015, 5(4), 1571-9.

[92] Chen, Y.; Li, L.; Zeng, J.; Wu, K.; Zhou, J.; Guo, P.; Zhang, D.; Xue, Y.; Liang, L.; Wang, X.; Chang, L.S.; He, D. Twist confers chemoresistance to anthracyclines in bladder cancer through upregulating P-glycoprotein. Chemotherapy, 2012, 58, $264 і ̈ 272$.

[93] Li, F.; Mahato, R.I. RNA interference for improving the outcome of islet transplantation. Adv. Drug Delivery Rev., 2011, 63(1і̄ 2), 47ī 68.

[94] Li, F.; Mahato, R.I. MicroRNAs and drug resistance in prostate cancers. Mol. Pharm., 2014, $11(8), 2539-52$. 
[95] Schneider, B.P.; Winer, E.P.; Foulkes, W.D.; Garber, J.; Perou, C.M.; Richardson, A. Triplenegative breast cancer: risk factors to potential targets. Clin. Cancer Res., 2008, 14, 8010ї 8018.

[96] Liedtke, C.; Mazouni, C.; Hess, K.R.; André, F.; Tordai, A.; Mejia, J.A. Response to neoadjuvant therapy and long-term survival in patients with triple-negative breast cancer. J. Clin. Oncol., 2008, 26, $1275 і ̈ 1281$.

[97] Wang, S.; Zhang, J.; Wang, Y.; Chen, M. Hyaluronic acid-coated PEI-PLGA nanoparticles mediated co-delivery of doxorubicin and miR-542-3p for triple negative breast cancer therapy. Nanomedicine, 2015, 12, 411-420.

[98] Bhargava-Shah, A.; Foygel, K.; Devulapally, R.; Paulmurugan, R. Orlistat and antisensemiRNA-loaded PLGA-PEG nanoparticles for enhanced triple negative breast cancer therapy. Nanomedicine, 2016, 11(3), 235-47.

[99] Shu, D.; Li, H.; Shu, Y.; Xiong, G.; Carson, W.E.; Haque, F.; Xu, R.; Guo, P. Systemic Delivery of Anti-miRNA for Suppression of Triple Negative Breast Cancer Utilizing RNA Nanotechnology. ACS Nano., 2015, 9(10), 9731-40.

[100] Wen, P.Y.; Kesari, S. Malignant gliomas in adults. N. Engl. J. Med., 2008, 359, 492 ï 507.

[101] Mamelak, A.N.; Jacoby, D.B. Targeted delivery of antitumoral therapy to glioma and other malignancies with synthetic chlorotoxin (TM-601). Expert Opin. Drug Deliv., 2007, 4, $175 \mathrm{I} 186$.

[102] Costa, P.M.; Cardoso, A.L.; Custódia, C.; Cunha, P.; Pereira de Almeida, L.; Pedroso de Lima, M.C. MiRNA-21 silencing mediated by tumor-targeted nanoparticles combined with sunitinib: A new multimodal gene therapy approach for glioblastoma. J. Control Release, 2015, 207, 31-9.

[103] Pazdur, R. National Cancer Institute at the National Institutes of Health. FDA Approval for Gemcitabine Hydrochloride. http://www.cancer.gov/cancertopics/druginfo/fda-gemcitabinehydrochloride (Accessed 2011). 
[104] Hung, S.W.; Marrache, S.; Cummins, S.; Bhutia, Y.D.; Mody, H.; Hooks, S.B.; Dhar, S.; Govindarajan, R. Defective hCNT1 transport contributes to gemcitabine chemoresistance in ovarian cancer subtypes: overcoming transport defects using a nanoparticle approach. Cancer Lett., 2015, $359(2), 233-40$.

[105] Muhammad, F.; Zhao, J.; Wang, N.; Guo, M.; Wang, A.; Chen, L.; Guo, Y.; Li, Q.; Zhu, G. Lethal drug combination: arsenic loaded multiple drug mesoporous silica for theranostic applications. Colloids Surf. B Biointerfaces, 2014, 123 506-14. 